Annals of Pure and Applied Mathematics

Vol. 16, No. 2, 2018, 307-312

ISSN: 2279-087X (P), 2279-0888(online)

Published on 18 February 2018

www.researchmathsci.org

DOI: http://dx.doi.org/10.22457/apam.v16n2a7

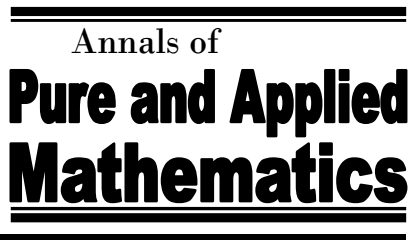

\title{
Prime Labeling to Drums Graphs
}

\author{
A.Edward Samuel ${ }^{1}$ and S. Kalaivani ${ }^{2}$ \\ Ramanujan Research Centre \\ PG and Research Department of Mathematics \\ Government Arts College (Autonomous) \\ Kumbakonam - 612 001, Tamilnadu, India \\ Bharathidasan University, Thiruchirappalli, India \\ e-mail: aedward74 thrc@yahoo.co.in; vanikalai.248@gmail.com \\ ${ }^{2}$ Corresponding author \\ Received 31 January 2018; accepted 13 February 2018
}

\begin{abstract}
In this paper, we introduce the new graph namely drums graph $D_{n}$. We investigate prime labeling for some graphs related to drums graph. We discuss prime labeling in the context of some graph operations namely duplication, fusion, switching in drums graph $D_{n}$. We prove that the duplication of any vertex of drums graph are prime graph. We prove that the identifying any vertex of drums graph are prime graph. We prove that the switching of an apex vertex and other vertex of drums graphs are prime graph and also apply coloring of the graph operations of the drums graph is satisfying coloring condition.
\end{abstract}

Keywords: Prime Labeling, Prime Graph, Drums Graph, Duplication, Fusion, Switching, coloring.

\section{AMS Mathematics Subject Classification (2010): $05 \mathrm{C} 78$}

\section{Introduction}

We consider only simple, finite, undirected and non - trivial graph $G=(V(G), E(G))$ with the vertex set $V(G)$ and the edge set $E(G)$. For notations and terminology we refer to Bondy and Murthy [8]. Many researchers have studied prime graph for example in $\mathrm{F}$ [7] have proved that the path $P_{n}$ on $n$ vertices is a prime graph. In Deretsky [11] have proved that the Cycle $C_{n}$ on $n$ vertices is a prime graph. For latest survey on graph labeling we refer to [9]. In Edward Samuel and Kalaivani [1] have proved the Prime labeling for some octopus related graphs. In Edward Samuel and Kalaivani [2] have proved the Prime labeling for some planter related graphs. In Edward Samuel and Kalaivani [3] have proved the Prime labeling for some vanessa related graphs. In Edward Samuel and Kalaivani [4] have proved the square sum labeling for some lilly related graphs. Sugumaran and Mohan [5] have discussed further results on prime cordial labeling. Sunoj and Mathew Varkey [6] have investigated the ADCSS-labeling for some middle graphs. Rajesh Kumar and Mathew Varkey [12] have discussed Gaussian neighborhood prime labeling of some classes of graphs and cycles. 


\section{A.Edward Samuel and S. Kalaivani}

\section{Preliminary definitions}

Definition 2.1. [11] Let $G=(V(G), E(G))$ be a graph with $p$ vertices. A bijection $f: V(G) \rightarrow\{1,2, \ldots, p\}$ is called a prime labeling if for each edge $e=u v, \operatorname{gcd}\{f(u), f(v)\}=1$. A graph which admits prime labeling is called a prime graph.

Definition 2.2. [2] Duplication of a vertex $v_{k}$ of a graph $\mathrm{G}$ produces a new graph $\mathrm{G}_{1}$ by adding a vertex $v_{k}{ }^{\prime}$ with $N\left(v_{k}{ }^{\prime}\right)=N\left(v_{k}\right)$. In other words a vertex $v_{k}{ }^{\prime}$ is said to be a duplication of $v_{k}$ if all the vertices which are adjacent to $v_{k}$ are now adjacent to $v_{k}{ }^{\prime}$ also.

Definition 2.3. [4] Let $u$ and $v$ be two distinct vertices of a graph $\mathrm{G}$. A new graph $\mathrm{G}_{1}$ is constructed by identifying(fusing) two vertices $u$ and $v$ by a single vertex $x$ is such that every edge which was incident with either $u$ or $v$ in $\mathrm{G}$ is now incident with $x$ in $\mathrm{G}_{1}$.

Definition 2.4. [4] A vertex switching $G_{v}$ of a graph $\mathrm{G}$ is obtained by taking a vertex $v$ of $\mathrm{G}$, removing all the entire edges incident with $v$ and adding edges joining $v$ to every vertex which are not adjacent to $v$ in $\mathrm{G}$.

Definition 2.5. [10] A $k$ - coloring of a graph $G=(V, E)$ is a function $c: V \rightarrow C$, where $|c|=k$. (Most often we use $c=[k]$ ). Vertices of the same color form a color class. A coloring is proper if adjacent vertices have different colors. A graph is $k$-colorable if there is a proper $k$-coloring. The chromatic number $\chi(G)$ of a graph $\mathrm{G}$ is the minimum $k$ such that $\mathrm{G}$ is $k$ - colorable.

\section{Prime labeling to drums graphs}

Drums graph 3.1. The Drums graph $D_{n}, n \geq 3$ can be constructed by two cycle graphs $2 C_{n}, n \geq 3$ joining two path graphs $2 P_{n}, n \geq 2$ with sharing a common vertex. i.e., $D_{n}=2 C_{n}+2 P_{n}$.

\section{Example 3.2.}

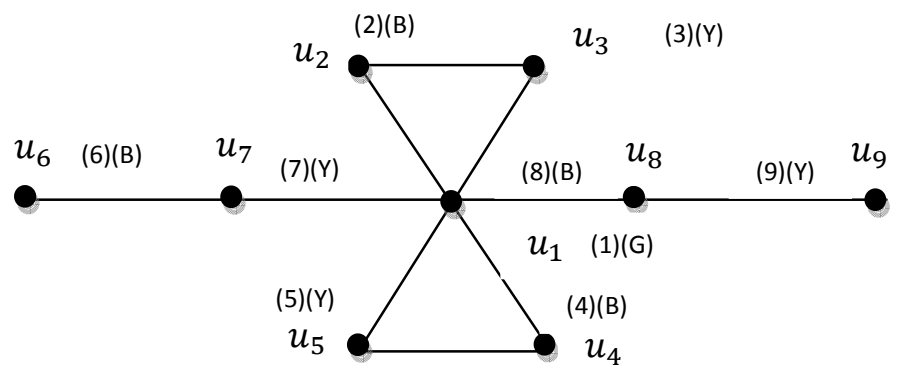

Figure 3.1: Drums graph $D_{3}$.

Theorem 3.3. The Drums graph $D_{n}, n \geq 3$ is a prime graph, where $n$ is any positive integer.

Proof: Let $D_{n}$ be the Drums graph with vertices $\left\{u_{1}, u_{2}, \ldots, u_{4 n-3}\right\}$. Here $\left|V\left(D_{n}\right)\right|=$ $4 n-3$, where $n$ is any positive integer.

Define a labeling $f: V\left(D_{n}\right) \rightarrow\{1,2, \ldots, 4 n-3\}$ as follows. 
Prime Labeling to Drums Graphs

$$
f\left(u_{i}\right)=i \text { for } 1 \leq i \leq 4 n-3
$$

Clearly vertex labels are distinct. Then for any edge $e=u_{1} u_{i} \in D_{n}$, $\operatorname{gcd}\left(f\left(u_{1}\right), f\left(u_{i}\right)\right)=\operatorname{gcd}\left(1, f\left(u_{i}\right)\right)=1$ and for any edge $e=u_{i} u_{i+1} \in D_{n}$, $\operatorname{gcd}\left(f\left(u_{i}\right), f\left(u_{i+1}\right)\right)=1$ for $2 \leq i \leq 4 n-3$ Since it is consecutive positive integers. Thus labeling defined above gives a prime labeling for a graph $D_{n}$. Thus $D_{n}$ is a prime graph.

\section{Example 3.4.}

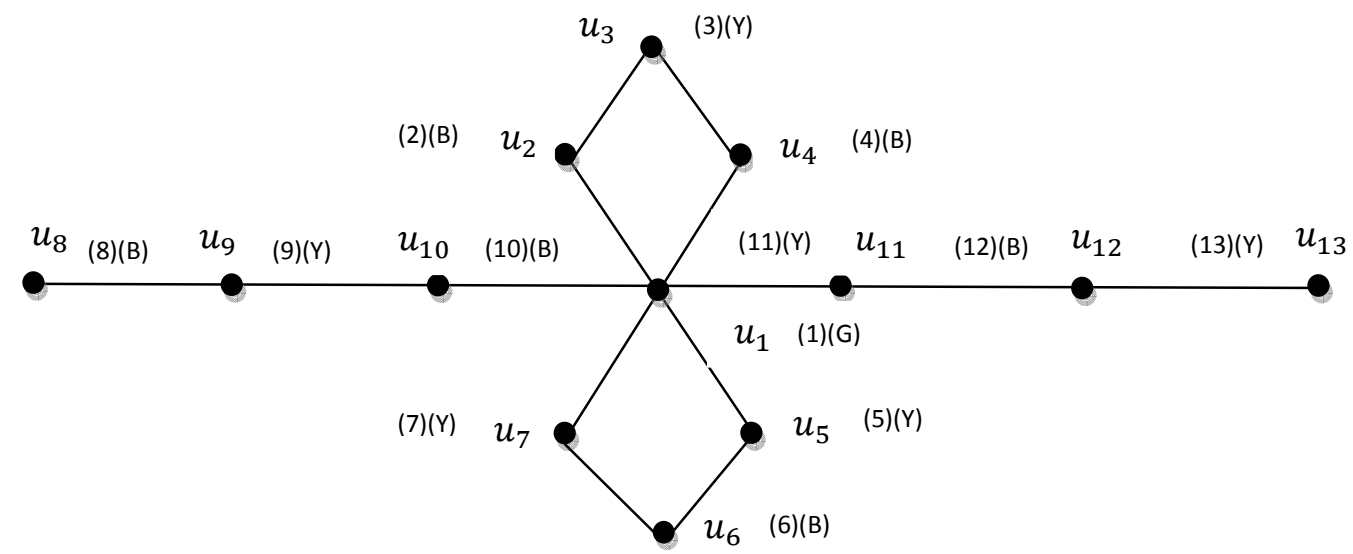

Figure 3.2: Prime labeling for $D_{4}$.

Theorem 3.5. The graph obtained by duplication of any vertex $u_{k}$ to $u_{k}{ }^{\prime}$ of drums graph $D_{n}, n \geq 3$ is a prime graph, where $n$ is any positive integer.

Proof: Let $\mathrm{G}$ be the graph of drums graph $D_{n}, n \geq 3$. Let $u_{k}$ be the vertex of the drums graph $D_{n}, n \geq 3$ and $u_{k}{ }^{\prime}$ be its duplicated vertex and $G_{k}$ be the graph resulted due to duplication of the vertex $u_{k}$ in $D_{n}, n \geq 3$, where $n$ is any positive integer. Let $V\left(D_{n}\right)=$ $\left\{u_{1}, u_{2}, \ldots, u_{4 n-3}\right\}$. Here $\left|V\left(G_{k}\right)\right|=4 n-2$.

We define a labeling $f: V\left(G_{k}\right) \rightarrow\{1,2, \ldots, 4 n-2\}$ as follows.

$$
\begin{gathered}
f\left(u_{i}\right)=i \quad \text { for } 1 \leq i \leq 2 n \\
f\left(u_{k}^{\prime}\right)=11, \quad f\left(u_{i}\right)=i+1 \text { for } 2 n+1 \leq i \leq 4 n-3
\end{gathered}
$$

Clearly vertex labels are distinct. Then $f$ admits prime labeling. Thus $G_{k}$ is a prime graph.

\section{Example 3.6.}

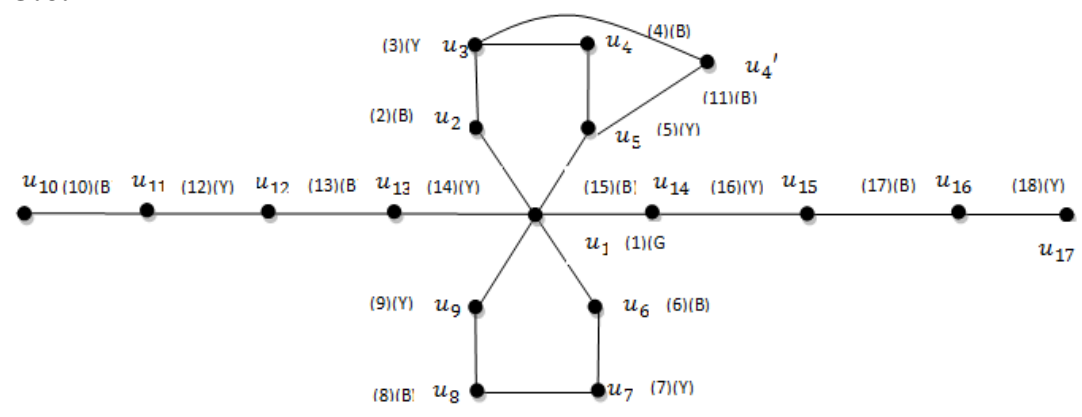

Figure 3.3: Duplication of $u_{4}$ in $D_{5}$. 


\section{A.Edward Samuel and S. Kalaivani}

Theorem 3.7. The graph obtained by identifying any two vertices $u_{i}$ and $u_{k}$ (where $d\left(u_{i}, u_{k}\right) \geq 3$ ) of drums graph $D_{n}, n \geq 3$ is a prime graph, where $n$ is any positive integer.

Proof: Let $D_{n}, n \geq 3$ be the drums graph with vertices $\left\{u_{1}, u_{2}, \ldots, u_{4 n-3}\right\}$ and the vertex $u_{i}$ be the fused with $u_{k}$. Denote the resultant graph as $G_{k}$. Here we note that $\left|V\left(G_{k}\right)\right|=$ $4 n-4$

Define a labeling $f: V\left(G_{k}\right) \rightarrow\{1,2, \ldots, 4 n-4\}$ as follows

$$
\begin{aligned}
& f\left(u_{i}\right)=i \quad \text { for } 1 \leq i \leq 4 n-5 \\
& f\left(u_{20}=u_{21}\right)=4 n-4
\end{aligned}
$$

Then $f$ admits prime labeling. According to this pattern the vertices are labeled such that for any edge $e=u_{i} u_{k} \in G_{k}, \operatorname{gcd}\left(f\left(u_{i}\right), f\left(u_{k}\right)\right)=1$.Clearly vertex labels are distinct. Thus we proved that the graph under consideration admits prime labeling. That is, the graph obtained by fusing (identifying) any two vertices $u_{i}$ and $u_{k}$ (where $d\left(u_{i}, u_{k}\right) \geq 3$ ) of drums graph $D_{n}, n \geq 3$ is a prime graph.

\section{Example 3.8.}

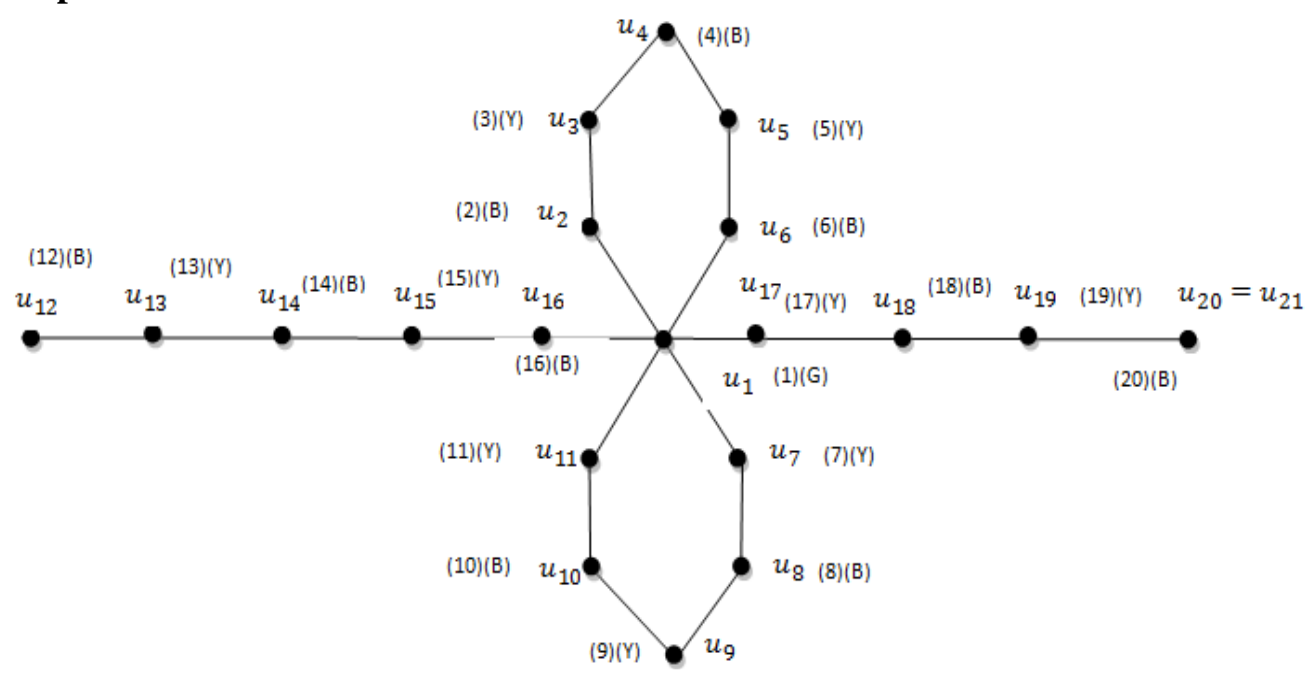

Figure 3.4: Fusion of $u_{20}$ and $u_{21}$ in $D_{6}$.

Theorem 3.9. The switching of any vertex $u_{k}$ in a drums graph $D_{n}, n \geq 3$ produces a Prime graph, where $n$ is any positive integer.

Proof: Let $G=D_{n}$ and $\left\{u_{1}, u_{2}, \ldots, u_{4 n-3}\right\}$ be the successive vertices of drums graph $D_{n}, n \geq 3$ and $G_{u}$ denotes the graph obtained by a vertex switching of $G$ with respect to the vertex $u$. It is obvious that $\left|V\left(G_{u}\right)\right|=4 n-3$.

Define a labeling $f: V\left(G_{u}\right) \rightarrow\{1,2, \ldots, 4 n-3\}$ as follows

$$
\begin{gathered}
f\left(u_{i}\right)=i \quad \text { for } 1 \leq i \leq n+1 \\
f\left(u_{i}\right)=i+1 \text { for } n+2 \leq i \leq 4 n-4 \\
f\left(u_{9}\right)=5
\end{gathered}
$$

Then for any edge $e=u_{i} u_{i+1} \in G_{u}, \operatorname{gcd}\left(f\left(u_{i}\right), f\left(u_{i+1}\right)\right)=1$ and for any edge $e=u_{1} u_{i} \in G_{u}, \operatorname{gcd}\left(f\left(u_{1}\right), f\left(u_{i}\right)\right)=\operatorname{gcd}\left(1, f\left(u_{i}\right)\right)=1$. Clearly vertex labels are 
Prime Labeling to Drums Graphs

distinct. Then $f$ admits prime labeling. Thus $G_{u}$ is a prime graph. That is, the switching of any vertex $u_{k}$ in a drums graph $D_{n}, n \geq 3$ produces a Prime graph, where $n$ is any positive integer.

Example 3.10.

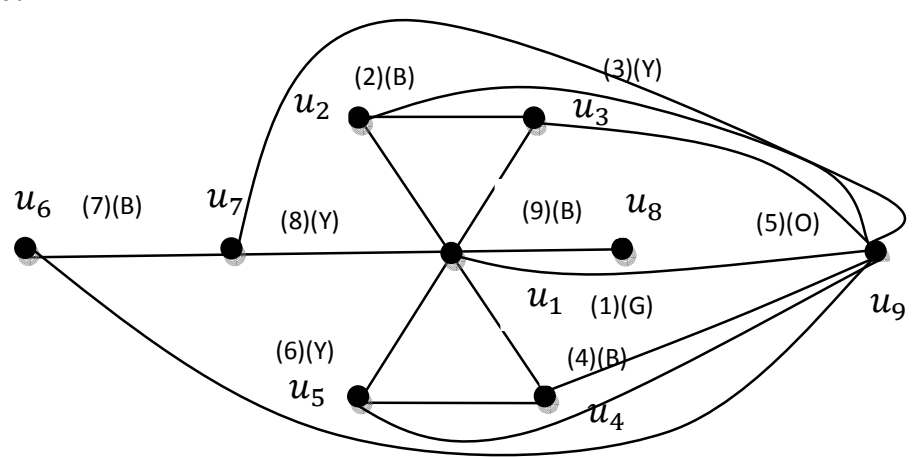

Figure 3.5: Switching the vertex $u_{9}$ in $D_{3}$.

Theorem 3.11. The switching of an apex vertex $u_{1}$ in a drums graph $D_{n}, n \geq 3$ produces a Prime graph, where $n$ is any positive integer.

Proof: Let $G=D_{n}$ and $\left\{u_{1}, u_{2}, \ldots, u_{4 n-3}\right\}$ be the successive vertices of drums graph $D_{n}, n \geq 3$ and $G_{u}$ denotes the graph obtained by an apex vertex switching of $G$ with respect to the vertex $u_{1}$. It is obvious that $\left|V\left(G_{u}\right)\right|=4 n-3$. Without loss of generality, we initiate the labeling from $u_{1}$ and proceed in the clock - wise direction.

Define a labeling $f: V\left(G_{u}\right) \rightarrow\{1,2, \ldots, 4 n-3\}$ as follows

$$
f\left(u_{i}\right)=i \text { for } 1 \leq i \leq 4 n-3
$$

Then for any edge $e=u_{i} u_{i+1} \in G_{u}, \operatorname{gcd}\left(f\left(u_{i}\right), f\left(u_{i+1}\right)\right)=1$ and for any edge $e=u_{1} u_{i} \in G_{u}, \operatorname{gcd}\left(f\left(u_{1}\right), f\left(u_{i}\right)\right)=\operatorname{gcd}\left(1, f\left(u_{i}\right)\right)=1$. Clearly vertex labels are distinct. Then $f$ is a prime labeling and consequently $G_{u}$ is a prime graph. That is, the switching of an apex vertex $u_{1}$ in a drums graph $D_{n}, n \geq 3$ produces a Prime graph and it is a disconnected graph.

\section{Example 3.12.}

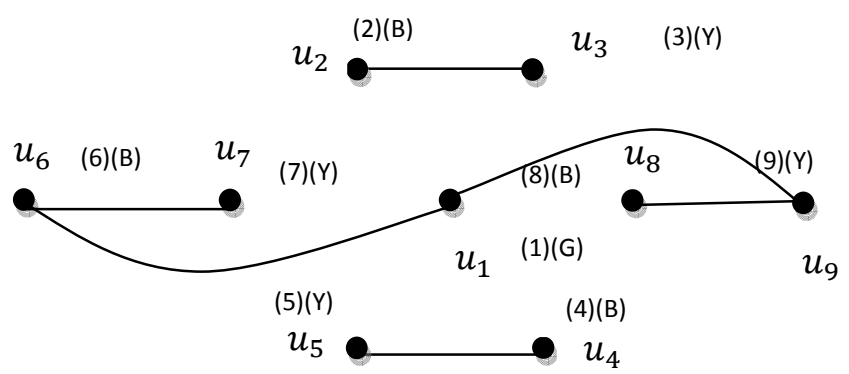

Figure 3.6: Switching an apex vertex $u_{1}$ in $D_{3}$

\section{Applications}




\section{A.Edward Samuel and S. Kalaivani}

The field of graph theory plays a vital role in various fields. One of the important areas in graph theory is graph labeling which is used in many applications like coding theory, radar, astronomy, circuit design, missile guidance, communication network addressing, $\mathrm{x}$ ray crystallography, data base management. Graph labeling is most useful to computer science like data mining, image processing, cryptography, software testing, information security, communication networks etc....

\section{Conclusion}

In this paper, we proved that the Drums graph $D_{n}$, duplication of the Drums graph $D_{n}$, fusing of the Drums graph $D_{n}$, switching of the Drums graph $D_{n}$ are prime graphs and also applied coloring condition to Drums graph $D_{n}$.

Acknowledgements. The authors are grateful to the referees whose valuable comments resulted in an improved paper.

\section{REFERENCES}

1. A. Edward Samuel and S. Kalaivani, Prime labeling for some octopus related graphs, IOSR Journal of Mathematics, 12(6) (2016) 57-64.

2. A. Edward Samuel and S. Kalaivani, Prime labeling for some planter related graphs, International Journal of Mathematics Research, 8(3) (2016) 221-231.

3. A. Edward Samuel and S. Kalaivani, Prime labeling for some vanessa related graphs, Indian Journal of Applied Research, 7(4) (2017) 136-145.

4. A. Edward Samuel and S. Kalaivani, Square sum labeling for some lilly related graphs, International Journal of Advanced Technology and Engineering Exploration, 4 (29) (2017) 68-72.

5. A.Sugumaran and V.Mohan, Further results on prime cordial labeling, Annals of Pure and Applied Mathematics, 14(3) (2017) 489-496.

6. B.S.Sunoj and T.K.Mathew Varkey, ADCSS-labeling for some middle graphs, Annals of Pure and Applied Mathematics, 12(2) (2016) 161-167.

7. H.C. Fu and K.C. Huany, On prime labeling, Discrete Math, 127 (1994) 181-186.

8. J.A. Bondy and U.S.R. Murthy, Graph Theory and Applications, North-Holland, Newyork, 1976.

9. J.A Gallian, A dynamic survey of graph labeling, The Electronic Journal of Combinations, 16 (2017) \# DS6.

10. R.L Brooks, On colouring the nodes of a network, Proc. Cambridge Phil. Soc. 37 (1941) 194-197.

11. T. Deretsky et.al, On vertex prime labeling of graphs in graph theory, Combinatories and applications, 1 (1991) 299-359.

12. T.J. Rajesh Kumar and T.K. Mathew Varkey, Gaussian neighborhood prime labeling of some classes of graphs and cycles, Annals of Pure and Applied Mathematics, 16(1) (2018) 133-140. 